\title{
UNC Lineberger Comprehensive Cancer
} Center

National Cancer Institute

\section{Source}

National Cancer Institute. UNC Lineberger Comprehensive Cancer Center. NCI

Thesaurus. Code C39554.

The mission of the UNC Lineberger Comprehensive Cancer Center is to reduce cancer occurrence and death through research, treatment, training, and outreach. It was designated as an $\mathrm{NCl}$ cancer center in 1975 and as a comprehensive cancer center in 1990. The center, which is affiliated with the University of North Carolina at Chapel Hill and UNC Cancer Care, has an emphasis on breast cancer research. 